\title{
A NOTE ON ABSOLUTE RIESZ SUMMABILITY FACTORS
}

\author{
HÜSEYIN BOR
}

Abstract. In the present paper a theorem on $\left|\bar{N}, p_{n}\right|_{k}$ summability factors of infinite series has been proved under more weaker conditions. Also we have obtained a new result concerning the $|C, 1|_{k}$ summability factors.

Mathematics subject classification (2000): 40D15, 40F05, 40G99.

Key words and phrases: absolute summability, almost increasing sequence.

\section{REFERENCES}

[1] S. Aljancic, D. ARAndelovic, O-regularly varying functions, Publ. Inst. Math., 22, (1977), 5-22.

[2] H. Bor, On two summability methods, Math. Proc. Camb. Philos Soc., 97, (1985), 147-149.

[3] H. Bor, A note on two summability methods, Proc. Amer. Math. Soc., 98, (1986), 81-84.

[4] H. Bor, A note on $\left|\bar{N}, p_{n}\right|_{k}$ summability factors of infinite series, Indian J. Pure Appl. Math., 18, (1987), 330-336.

[5] H. BoR, Absolute summability factors for infinite series, Indian J. Pure Appl. Math., 19, (1988), 664-671.

[6] T. M. FleTt, On an extension of absolute summability and some theorems of Littlewood and Paley, Proc. London Math. Soc., 7, (1957), 113-141.

[7] G. H. Hardy, Divergent Series, Oxford Univ. Press., Oxford, (1949).

[8] E. KogBetLiantz, Sur les séries absolument sommables par la méthode des moyennes arithmétiques, Bull. Sci. Math., 49, (1925), 234-256.

[9] S. M. MAZHAR, A note on absolute summability factors, Bull. Inst. Math. Acad. Sinica, 25, (1997), 233-242.

[10] K. N. Mishra, R. S. L. SRIVAStaVA, On $\left|\bar{N}, p_{n}\right|$ summability factors of infinite series, Indian J. Pure Appl. Math., 15, (1984), 651-656. 